\title{
Advancing neuroimaging research in South Africa
}

Neuropsychiatric disorders account for the second-largest proportion of the burden of disease in South Africa. ${ }^{1}$ This fact places South African clinical and research neuroscientists in an often unique position to explore critical questions that are of international relevance. The use of high field strength MRI, a particularly versatile imaging tool, was conceptualised to address the lack of opportunity and capacity to do this kind of work in the South African context. As such, a multi-modality brain imaging initiative in the Western Cape, with MRI at the centre, evolved in 2007 to create a world-class imaging research and training environment. With the establishment of the Cape Universities Brain Imaging Centre (CUBIC), a unique opportunity has been created for research and training in a multidisciplinary model that includes strong input from the radiology sector.

CUBIC represents a joint initiative between the Universities of Stellenbosch and Cape Town, and Siemens. The primary focus of the centre is on neuroscience research. The CUBIC houses a Siemens Magnetom Allegra - a head-only 3T MRI scanner which is currently one of the most advanced brain imaging instruments available. Located on the Stellenbosch University Health Sciences campus, CUBIC was commissioned in March 2007. In addition to being the only Siemens Allegra MRI scanner on the African continent, CUBIC is also South Africa’s best-equipped functional MRI (fMRI) facility, housing dedicated electroencephalography and near-infrared spectroscopy research laboratories.

From being staffed and supported by highly qualified imaging and engineering experts, the first 18 months of operation of the centre has already lead to the development of in-house tools and expertise for a wealth of advanced MRI techniques including fMRI, diffusion tensor imaging (DTI), white matter fibre tractography, multi-voxel MR spectroscopy, 3D cerebrospinal fluid flow quantification, and localised anatomical 3D volumetric measurements. The $3 \mathrm{~T}$ facility combined with a rapidly advancing image analysis field, allows researchers to use highspeed imaging capacity at $3 \mathrm{~T}$ in modalities that help to ask increasingly specific questions.

Through a variety of both local and international collaborations and research grants, numerous research projects are being undertaken at CUBIC. Their operational success relies largely on the involvement of graduate students from a range of disciplines that includes radiology, psychiatry, psychology and biomedical engineering. In line with the burden of disease studies and Medical Research Council research priorities, research covers clinical areas such as substance abuse, fetal alcohol syndrome, tuberculous meningitis and HIV-associated cognitive impairment. Numerous students are involved in technology-based projects which include custom MRI coil design, developing specialised data post-processing tools, and MRI pulse sequence development.

The CUBIC offers a limited clinical service to public sector patients in need of MRI brain scans. This outreach opportunity has enabled local departments of radiology to enhance the training of registrars/residents in radiology by exposing them to high field imaging.

The facility also provides unique opportunities for the South African radiological community. Mastering the post-processing tools for the advanced neuro-MRI techniques is often impractical in a clinical setting. We believe that there is a niche need for tailored image acquisition, post-processing and interpretation. A battery of tools can be combined for both diagnostic and neurosurgical planning purposes. For example: metabolite concentrations can be mapped in and around a tumour using chemical shift imaging; the integrity and trajectory of important white matter fibre bundles can be assessed using DTI fibre tracking; and the position of functional brain regions relative to a tumour can be accurately localised using fMRI.

The CUBIC has gathered significant momentum in a relatively short time. We have already demonstrated the utility of high field strength MRI in our clinical environment. The growing interest of students in brain imaging is already a welcome sign that this opportunity is overdue. MRI technology has been advancing at a formidable pace, and research scanners are becoming commonplace at universities across the globe. We hope to extend this facility in time to full-body MRI research, tailored to the uniquely South African disease burden.

See www.sun.ac.za/cubic for more details about the Cape Universities Brain Imaging Centre.

\section{B S Spottiswoode, P D Carey}

Cape Universities Brain Imaging Centre, Faculty of Health Sciences, Stellenbosch University and University of Cape Town

Stein DJ, Daniels W, Emsley R et al. A brain-behaviour initiative for South Africa: the time is right. Metab Brain Dis 2006; 21(2-3): 279-294. 